\title{
HIGH CAPACITY COUNTER CURRENT CHROMATOGRAPHY FOR THE ISOLATION OF ACTIVE INGREDIENTS FROM MEDICINAL PLANTS
}

\section{(C) Ignatova Svetlana}

Advanced Bioprocessing Centre, Brunel Institute for Bioengineering, Brunel University West London, Uxbridge, UB83PH, UK

Countercurrent chromatography (CCC) is a liquidliquid purification technology with both liquid mobile and stationary phases. Being a solid support free technique, it has many advantages for the isolation of active ingredients from medicinal plant materials such as handling crude extracts including particulates, no irreversible adsorption and therefore, $100 \%$ sample recovery. CCC is being widely used across the world for natural product research, especially in China, Korea, Brazil, South Africa, Singapore, Tasmania and Europe. At Brunel Institute for Bioengineering and its Advanced Bioprocessing Centre, we have developed, in partnership with Dynamic Extraction Ltd, three scales of high performance high throughput CCC-centrifuges: MINI (5-40ml), MIDI (39-1L) and MAXI (4.6 and 18L). Each is capable of giving separations in the order of minutes. Purifications are optimised on the MINI with minimum crude and solvents used and then scaled to the MIDI or MAXI with no loss of resolution and the same fast processing time, while throughputs can be between $1-5 \mathrm{~kg} /$ day. CCC technology is being used to isolate active principles from medicinal plant extracts in collaboration with Sichuan University, Tsinghua University, Shanghai East China University and Guangzhou Xiangxue Pharmaceutical Co. Several case studies and latest research on CCC applications will be presented. Being part of European Framework 7 "Good Practice in Traditional Chinese Medicine Research" Consortium we take an active part in developing easy-to-follow statements on the various regulatory frameworks for complex herbal mixtures to facilitate commercialisation of Chinese Herbal Medicines in Western markets. CCC plays an important role in the quality control and standardisation process of TCM as one of the reliable extraction and separation technologies.

\section{SCHIZANDRA OIL EXTRACT IS A SOURCE OF ANTIOXIDANT COMPOUNDS}

\section{() Ivanova S. A., Pozharitskaya O. N., Demchenko D. V., Makarov V. G.}

\section{St-Petersburg Institute of Pharmacy, Russia}

The aim of this study was to determine schizandra oil extract free radical scavenger activity (FRSA) and its association with the extract composition. Schizandra chinensis oil extract was used. Test solution was prepared by methanol extraction $(2 / 1 \mathrm{v} / \mathrm{v})$. The DPPH method was used for estimating FRSA of the test solution by spectrophotometry
(1) and by HPTLC-DPPH • (2). A samples were spotted on Silica gel $60 \mathrm{~F}$ 254s glass plates (Merck, Germany) using a Linomat V (Camag). The plates were developed in mixture of toluene/ethylacetate (7/3). Plates detection was carried out with TLC Scanner 3 (Camag). IC50 of Schizanda oil extract was established $2.9 \mathrm{mg} / \mathrm{ml}$ by spectrophotometry.

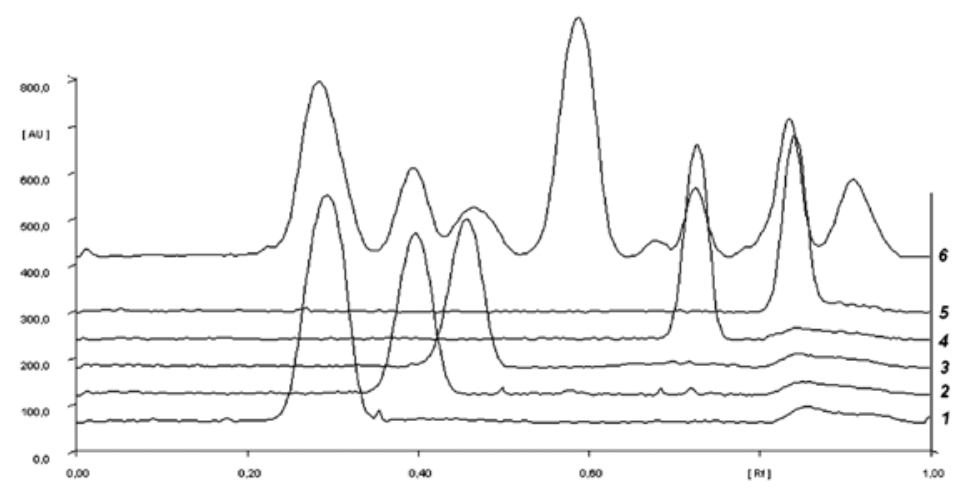

A)

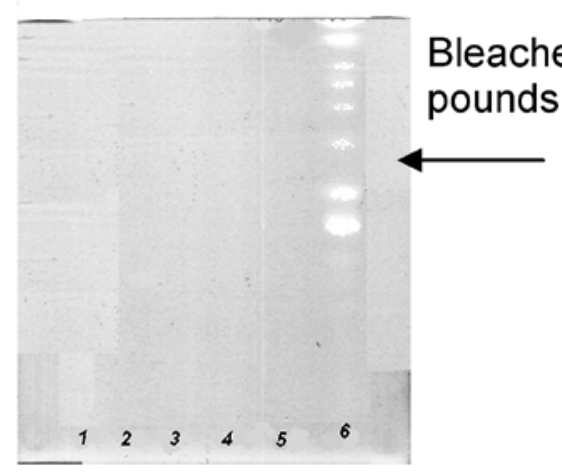

B)

Figure1. Densitogramms by $254 \mathrm{~nm}(\mathrm{~A})$ and derivatized by DPPH solution plate in day-light (B). 1-5 - schisandrols $A$ and $B$, schisantherin $A$, schisandrins $A$ and $B$ respectively (all Phytolab, $1 \mathrm{mg} / \mathrm{ml}$ methanol, $2 \mu \mathrm{l}$ ); 6 - test solution, $5 \mu \mathrm{l}$ 
Schisandrols A and B, schisantherin A, schisandrins A and $B$ were detected in Schisandra oil extract by HPTLC (fig. 1A). These lignans are considered to be the main active substances of Schizandra chinensis. Lignans antioxidant activity plays a significant role in pharmacological activity of Schizandra drugs (3). We established that these lignans don't have FRSA by DPPH. They don't bleach background of the plate after DPPH solution derivatization (fig. 1B). FRSA of Schizandra oil extract depend on other components of the extract, but not lignans.
Thus, Schizandra oil extract is a source not only of the well-known lignans, but of other active compounds with the FRSA. The FRSA of these substances causes of their pharmacological importance.

References: (1) MA Gyamfi, M Yonamine, Y Aniya (1999). Gen Pharmacol., 32: 661-667. (2) ON Pozharitskaya, SA Ivanova, AN Shikov, VG Makarov (2007). J Sep Sci., 30: 1250-1254. (3) A Panossian, G Wikman (2008). J. Ethnopharmacology, 118: 183-212.

\title{
POTENTIAL OF PUERARIA CANDOLLEI VAR. MIRIFICA AND MIROESTROL ON ANTIOXIDANT ENZYMES IN UTERI OF OVARIECTOMIZED MICE
}

\section{(C) Jarukamjorn Kanokwan ${ }^{1,2}$, Chatuphonprasert Waranya ${ }^{1,2}$, Montakantirat Orawan $^{2}$, Putalun Waraporn ${ }^{1,2}$, Chaichantipyuth Chaiyo ${ }^{3}$}

\author{
${ }^{1}$ Research Group for Pharmaceutical Activities of Natural Products using Pharmaceutical Biotechnology (PANPB), National \\ Research University, Khon Kaen University, Thailand \\ ${ }^{2}$ Faculty of Pharmaceutical Sciences, Khon Kaen University, Thailand \\ ${ }^{3}$ Faculty of Pharmaceutical Sciences, Chulalongkorn University, Bangkok, Thailand
}

Pueraria candollei Wall. ex Benth. var. mirifica (PM; family Leguminosae) has long been used in Thai traditional medicine for rejuvenation. Reactive oxygen species lead to cellular damage when rate of generation exceeds rate of decomposition by antioxidant defense systems, i.e., glutathione peroxidase (GPx), catalase (CAT), superoxide dismutase (SOD), and reduced glutathione (GSH). The present study aims to evaluate potential of PM and its strong phytoestrogen miroestrol (MR) on antioxidant enzymes in uteri of ovariectomized (OVX) mice. Adult OVX ICR mice were daily given estradiol benzoate (E2), the PM crude extract, or MR, for $2 \mathrm{~m}$. The uteri were collected at $24 \mathrm{~h}$ after the last treatment to measure the levels of antioxidant species $(1,2)$. OVX lowered the levels of GPx, CAT, SOD, and GSH in the uteri. Though E2 did not improve antioxidant enzymes to the normal levels, it extensively elevated the ratio of
GSSG/GSH in the OVX uteri. PM and MR significantly recovered the levels of GPx, CAT, and SOD in the OVX with increasing the ratio of GSSG/GSH to the levels comparable to the normal levels. These observations revealed, for the first time, antioxidant potentials of PM and MR via increasing the levels of antioxidant related enzymes and GSH species, resulted in improving antioxidant defense status.

References: (1) Ozmen, B., Ozmen, D., Erkin, E., Guner, I., Habif, S., Bayındır, O., 2002. Lens superoxide dismutase and catalase activities in diabetic cataract. Clin. Biochem. 35: 69-82. (2) Pinto, R.E., Bartley, W., 1969. The effect of age and sex on glutathione reductase and glutathione peroxidase activities and on aerobic glutathione oxidation in rat liver homogenates. Biochem. J. 112: 109-115. 